\title{
Infrared Spectroscopy as a Probe of Electronic Energy Transfer
}

\author{
Valeriu Scutelnic, ${ }^{\dagger}$ Antonio Prlj, ${ }^{\ddagger}$ Aleksandra Zabuga, ${ }^{\dagger}$ Clémence Corminboeuf, $^{\ddagger}$ \\ and Thomas R. Rizzo* ${ }^{*}+$ (1) \\ ${ }^{\dagger}$ Laboratory of Molecular Physical Chemistry, Ecole Polytechnique Fédérale de Lausanne, Station 6, CH-1015 Lausanne, Switzerland \\ ${ }^{\ddagger}$ Laboratory for Computational Molecular Design, Ecole Polytechnique Fédérale de Lausanne, CH-1015 Lausanne, Switzerland
}

\section{Supporting Information}

\begin{abstract}
We have combined electronic and vibrational spectroscopy in a cryogenic ion trap to produce highly resolved, conformer-selective spectra for the ground and excited states of a peptide containing two chromophores. These spectra permit us to determine the precise three-dimensional structure of the peptide and give insight into the migration of the electronic excitation from phenylalanine to tyrosine because changes in the excited-state infrared spectra are sensitive to localization of the electronic energy in each chromophore. The well-controlled experimental conditions make this result a stringent test for theoretical methods dealing with electronic energy transfer.
\end{abstract}

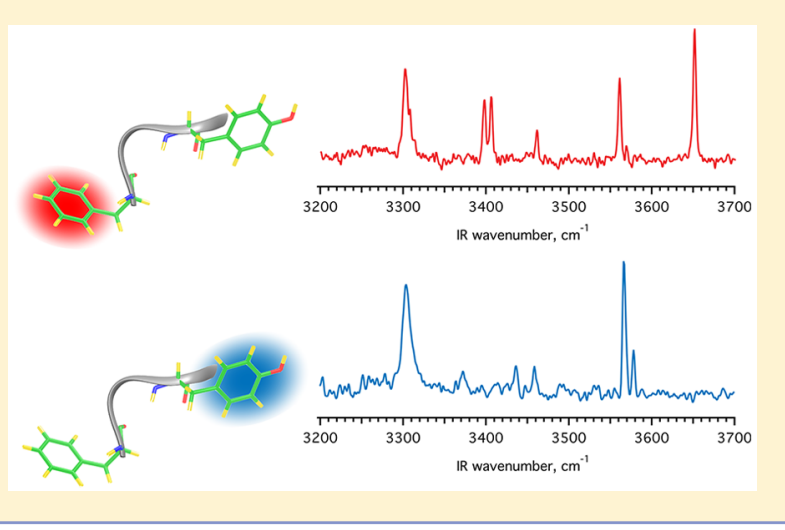

7 lectronic energy transfer (EET) processes are ubiquitous in nature; for instance, they play a central role in photosynthesis $^{1}$ and photolyase activity. ${ }^{2}$ Förster resonance energy transfer (FRET) is widely used in structural biology to measure the distance between donor and acceptor chromophores in proteins. ${ }^{3}$ Moreover, accurate modeling of EET could assist in the engineering of more efficient solar cells. ${ }^{4,5}$ While the majority of EET studies are performed in solution at room temperature, theoretical modeling of EET would significantly benefit from experiments carried out on isolated molecules at low temperature, which would allow for conformer-specific measurements. This is essential because both the distance and orientation of the chromophores play key roles in the energy transfer efficiency.

Conformation-dependent EET in the gas phase was first demonstrated in molecular beam experiments by Chattoraj et al. ${ }^{6}$ More recently, other research groups have extended EET studies to gas-phase ions inside of a mass spectrometer. ${ }^{7-9}$ However, fluorescence detection from ions stored in an ion trap is challenging, owing to the low chromophore density and restricted angle for photon collection. Dugourd and co-workers introduced an "action-FRET" technique ${ }^{10}$ that circumvents these obstacles by measuring the EET efficiency by means of specific photofragmentation rather than by fluorescence. However, a detailed picture of the energy dissipation processes that leads to fragmentation is still lacking.

The objective of this work is to measure EET rates of a gasphase peptide of well-defined geometry in a cryogenic ion trap. We use infrared-ultraviolet (IR-UV) double resonance ${ }^{11}$ to obtain a ground-state infrared (IR) spectrum of each conformer as well as to determine its contribution to the electronic spectrum. By comparing these highly resolved IR spectra with those computed for the lowest-energy conformers determined by theory, we can define the distance between the chromophores and their relative orientation. We then use a UV laser pulse to promote a single conformer to the excited state of a specific chromophore. An IR laser then probes the electronically excited molecules, producing a unique spectral fingerprint that is characteristic of each electronic state. Monitoring specific IR transitions as a function of the delay time between pump and probe pulses provides a measure of the excited-state lifetimes and hence the absolute rates of EET.

For this we chose a model peptide, Ac-FAYK- $\mathrm{H}^{+}$. The photochemistry of short peptides containing phenylalanine or tyrosine chromophores has been extensively characterized, ${ }^{12-18}$ making Ac-FAYK- $\mathrm{H}^{+}$an ideal system in which to study EET. The C-terminal lysine side-chain induces strong hydrogen bonds with the backbone carbonyls, ${ }^{19}$ stabilizing the peptide in a structure similar to the capping motif ${ }^{20}$ of a $3_{10}$ helix. $^{21}$ This provides a well-defined scaffold for the two chromophores involved. This is not unlike the work of Hendricks et al., who used the polyalanine helical motif to evaluate the EET efficiency from tryptophan to a disulfide bond; ${ }^{22}$ however, their studies were not conformer-selective. The controlled conditions of our experimental approach provide a stringent test for models of EET.

To begin, we characterize the UV spectroscopy of the peptide of interest. Upon electronic excitation of the cold AcFAYK $-\mathrm{H}^{+}$with a UV laser, a fraction of ions dissociates. Scanning the UV laser and monitoring the ion fragment signal

Received: April 18, 2018

Accepted: May 31, 2018

Published: May 31, 2018 


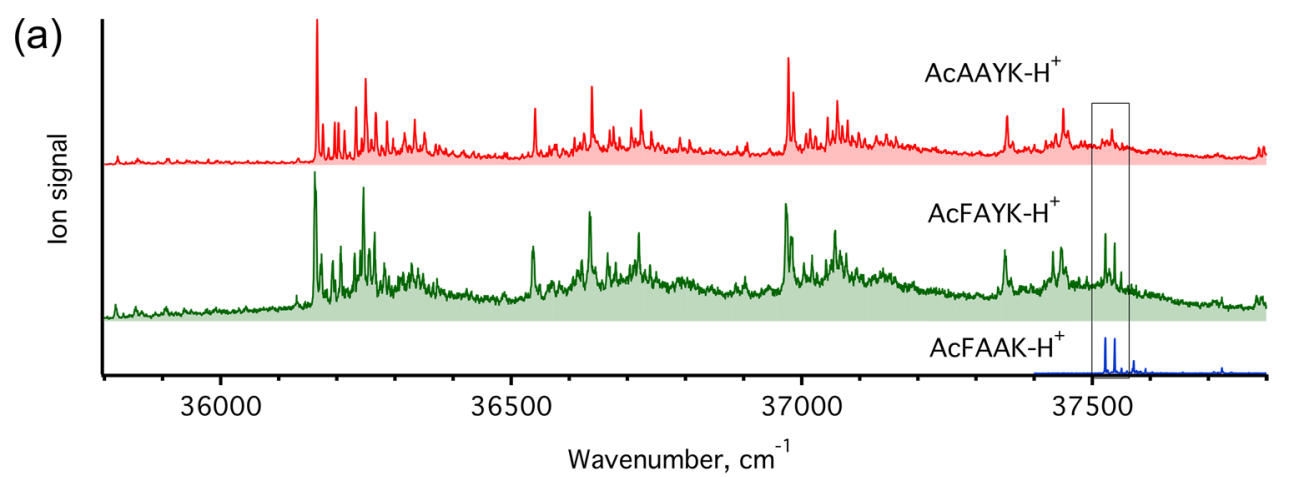

(b)

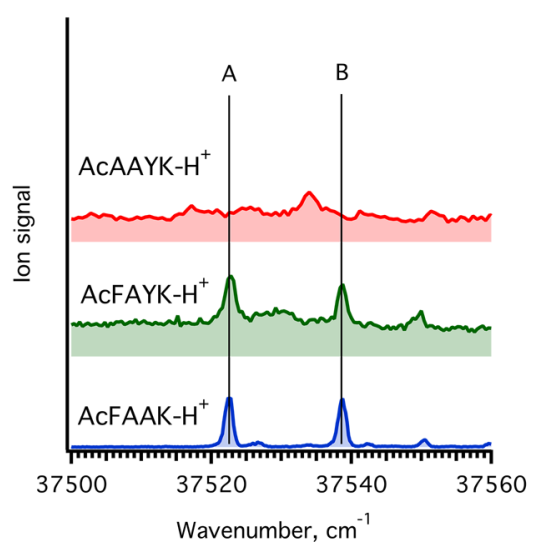

Figure 1. (a) Electronic fragmentation spectrum of AcAAYK- $H^{+}$(red), AcFAYK-H ${ }^{+}$(green), and AcFAAK-H ${ }^{+}$(blue). (b) Blow-up of the rectangular slice. Vertical lines indicate electronic transitions of the two major conformers A and B.

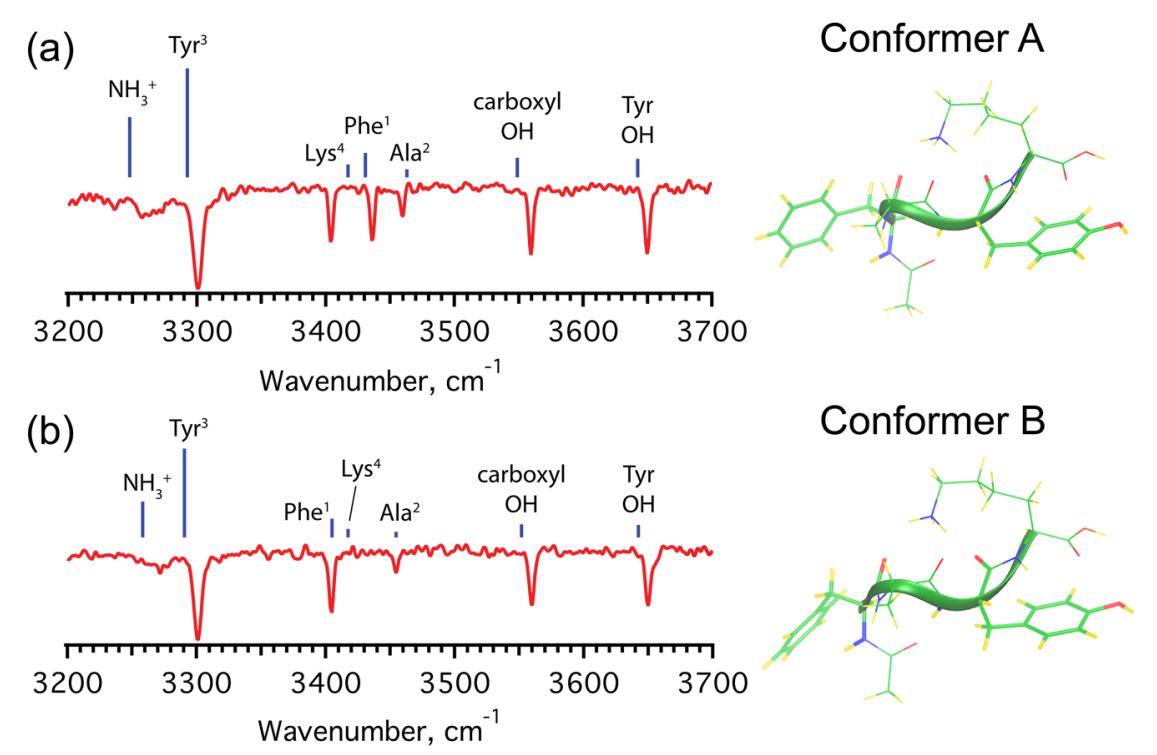

Figure 2. Measured IR spectra of the conformers of AcFAYK- $\mathrm{H}^{+}$that exhibit electronic transitions at (a) $37522.8 \mathrm{~cm}^{-1}$ (conformer A) and (b) $37538.8 \mathrm{~cm}^{-1}$ (conformer B) along with the theoretical IR spectra (blue lines) and the corresponding calculated structure. Assignments of the lines are made based on the computed vibrational spectra, the details of which are found in the Supporting Information.

generates an electronic action spectrum of AcFAYK-H $\mathrm{H}^{+}$(Figure la, green trace).

Although the UV spectrum of AcFAYK- $\mathrm{H}^{+}$is congested due to the high Franck-Condon activity of the tyrosine chromophore, ${ }^{23,24}$ the sharp band at $37522.8 \mathrm{~cm}^{-1}$ can be assigned to the band origin of the phenylalanine chromophore absorption. In addition to being close in wavenumber to the band origin of protonated phenylalanine, this assignment is confirmed by comparison with the electronic spectrum of a peptide in which tyrosine was replaced by alanine (AcFAAK$\mathrm{H}^{+}$, blue spectrum in Figure $1 \mathrm{~b}$ ). The nearly identical positions of the phenylalanine vibronic transitions in AcFAYK- $\mathrm{H}^{+}$and AcFAAK- $\mathrm{H}^{+}$imply that the two peptides have similar structure, the confirmation of which is discussed below. Moreover, the $\mathrm{UV}$ spectrum of AcFAYK- $\mathrm{H}^{+}$resembles that of AcAAYK- $\mathrm{H}^{+}$ (red trace in Figure 1a) to a surprisingly high degree, also implying a similar structure. Electronic transitions at 37522.8 and $37538.8 \mathrm{~cm}^{-1}$, highlighted in Figure $1 \mathrm{~b}$, belong to the two 

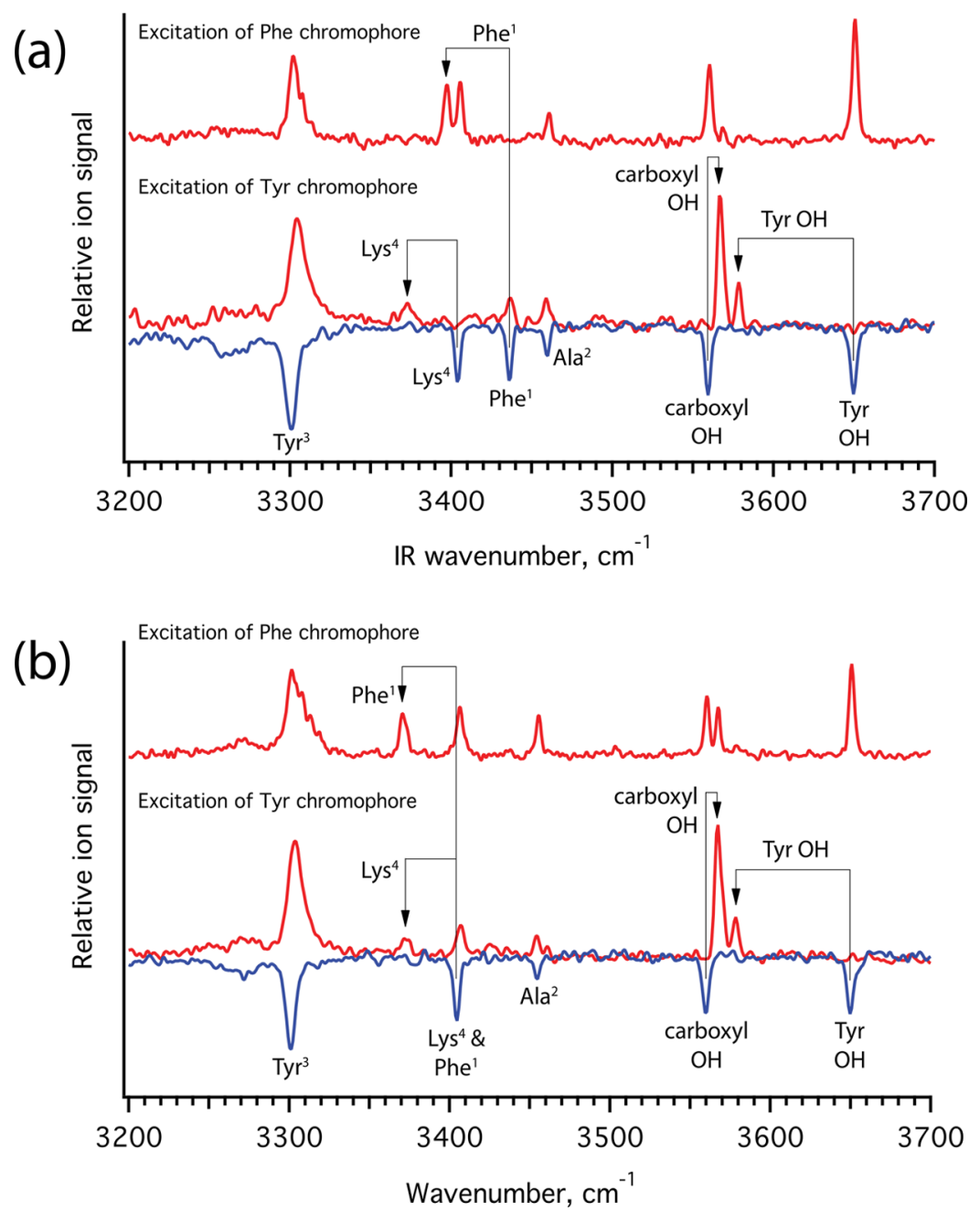

Figure 3. (a) IR spectra of the excited states of conformer A (red) with the electronic excitation localized in the phenylalanine and tyrosine chromophores compared with the ground state (blue). The UV laser is fixed at 37522.8 and $36193 \mathrm{~cm}^{-1}$ for pumping the phenylalanine and tyrosine chromophore, respectively. (b) For conformer B, the phenylalanine and tyrosine chromophore transitions occur at 37538.8 and $36197 \mathrm{~cm}^{-1}$, respectively.

dominant conformers of AcFAYK- $\mathrm{H}^{+}$, denoted as A and $\mathrm{B}$. We measure an infrared spectrum for each peak in the phenylalanine absorption region of AcFAYK- $\mathrm{H}^{+}$by applying IR-UV double resonance, shown in Figure 2 along with the computed spectra and corresponding structures. The good agreement between measured and computed spectra allow us to assign the structures of the two conformers. Comparison of the AcFAYK$\mathrm{H}^{+}$IR spectra with those of the monochromophoric peptides reveals similar features arising from the same hydrogen-bonding pattern; the difference is exclusively due to weak perturbation of the $\mathrm{NH}$ and $\mathrm{OH}$ groups in the vicinity of phenylalanine or tyrosine chromophores (see Figure S1).

Analysis of the structures shows that the only difference between conformers $\mathrm{A}$ and $\mathrm{B}$ consists of a $120^{\circ}$ rotation of the Phe side-chain around the $\mathrm{C}_{\alpha}-\mathrm{C}_{\beta}$ bond (Figure S2). In conformer $\mathrm{B}$, this difference allows the $\mathrm{Phe}^{1}$ amide $\mathrm{NH}$ to form a favorable $\pi$-hydrogen bond with the benzene ring, and this interaction shifts one band from 3436 to $3404 \mathrm{~cm}^{-1}$. The presence of two conformers that differ only by the phenylalanine side-chain flip is a common property of $3_{10}$ helices with phenylalanine at the $\mathrm{N}$-terminus..$^{20,25,26}$ It is essential to stress that the distance between the chromophores' centers remains virtually the same (11.7 and $11.3 \AA$ for conformers $\mathrm{A}$ and B, respectively); only the relative orientation of the chromophores changes substantially.

Excited-state infrared spectra are measured by fixing the UV laser to a strong transition of either the tyrosine chromophore at $36193 \mathrm{~cm}^{-1}$ or the phenylalanine chromophore at 37522.8 $\mathrm{cm}^{-1}$, related to conformer A and scanning the IR frequency. Correction for the tyrosine absorption under the sharp phenylalanine bands is done by a subtraction procedure described in the Supporting Information. The gain spectra (Figure 3a, shown in red) are recorded at the shortest ( $\sim 5 \mathrm{~ns}$ ) delay between the UV pump and IR probe lasers.

The excited-state vibrational spectra are similar to the corresponding ground-state spectra, as only certain bands undergo a noticeable shift relative to their frequency in the ground state. The infrared spectrum of conformer $\mathrm{A}$ in the excited electronic state is different depending on which chromophore is excited. The peak assignments of the ground-state IR spectrum allow us to determine which $\mathrm{NH}$ and $\mathrm{OH}$ groups experience a frequency shift in the excited state. Thus, excitation of the phenylalanine chromophore shifts only the $\mathrm{Phe}^{1} \mathrm{NH}$ line by $39 \mathrm{~cm}^{-1}$ from its ground-state frequency. This indicates that the electronic energy is localized on the phenylalanine chromophore and not on the tyrosine. Excitation 

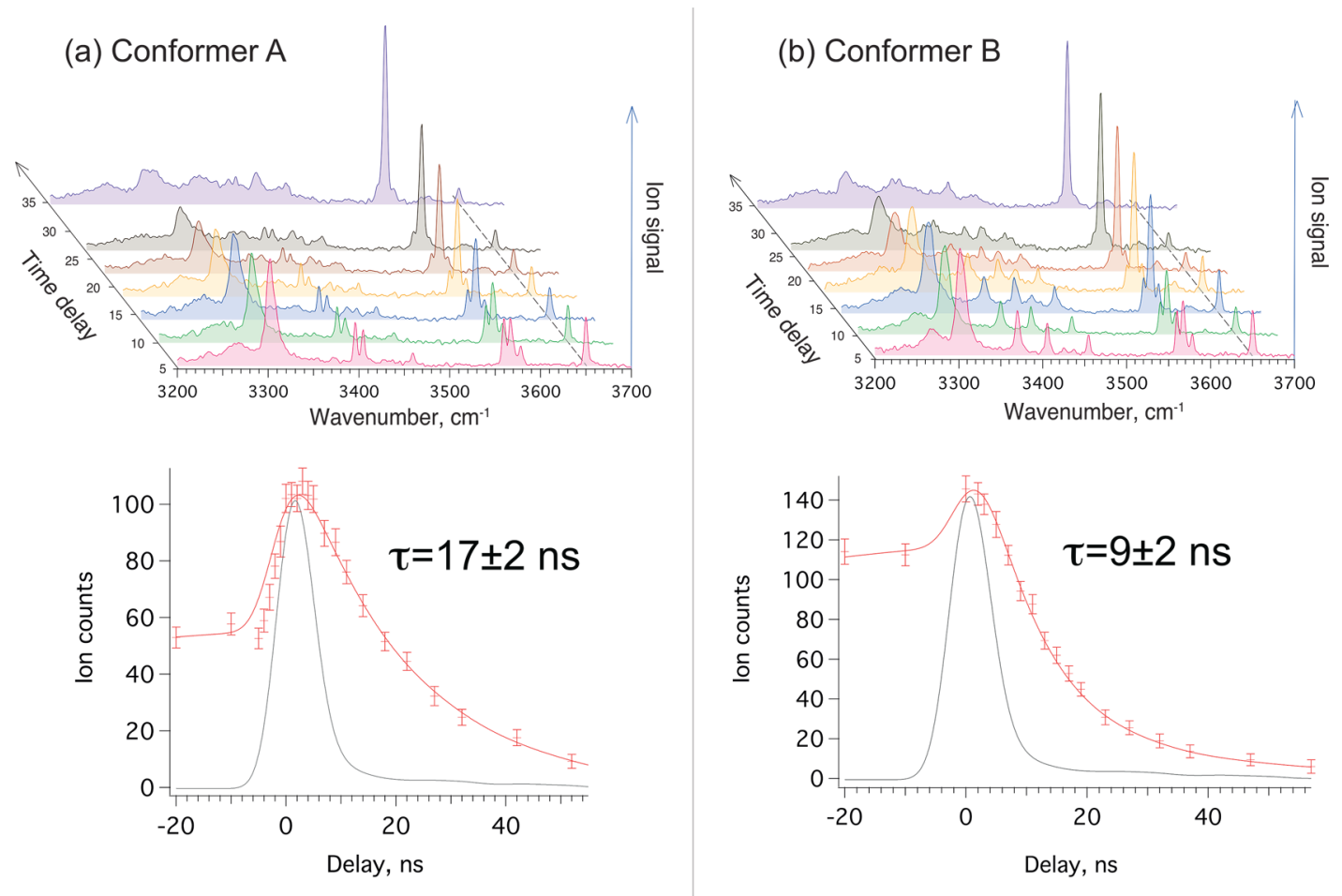

Figure 4. (Top) Time-resolved IR spectra. (Bottom) Transient signal (dots with error bars) of the singlet excited state of the phenylalanine chromophore, representing a cut of the $3 \mathrm{D}$ plots along the dashed lines. The cross correlation of the two laser pulses is depicted in gray, and the solid red lines are convolutions of the cross-correlation signal with theoretical decays. Absorption of the conformers A and B in their ground state at 3650 $\mathrm{cm}^{-1}$ causes positive signals at negative delays.

of the tyrosine chromophore results in shifts of other bands: the tyrosine $\mathrm{OH}$ appears red-shifted by $71 \mathrm{~cm}^{-1}$ (similar to the 76 $\mathrm{cm}^{-1}$ red shift of $\mathrm{OH}$ upon $\mathrm{S}_{0}-\mathrm{S}_{1}$ excitation of phenol ${ }^{27}$ ); the carboxyl $\mathrm{OH}$ is blue-shifted by $7 \mathrm{~cm}^{-1}$; and the Lys ${ }^{4} \mathrm{NH}$ band is red-shifted by $32 \mathrm{~cm}^{-1}$. These groups are in close proximity to the tyrosine chromophore, suggesting that the perturbation of the electronic structure in this state is restricted exclusively around tyrosine. Analogous changes in the IR spectra of conformer B occur upon electronic excitation (Figure $3 b$ ): the overlapping $\mathrm{Lys}^{4}$ and $\mathrm{Phe}^{1}$ bands are separated upon excitation of the phenylalanine chromophore, $\mathrm{Phe}^{1}$ shifting to the red by $34 \mathrm{~cm}^{-1}$ (consistent with reference ${ }^{14}$ ). Quantum chemical computations for AcFAYK- $\mathrm{H}^{+}$(see computational details in the SI, Figures S4 and S5) show that the first and second excitations correspond to $\mathrm{L}_{\mathrm{b}}$ type transitions ${ }^{28}$ of the tyrosine and phenylalanine chromophores, respectively. The localized character of the excitations explains why the groups close to the chromophore experience the aforementioned shift in frequency. Moreover, the excited-state spectra are sharp, indicating that the ions in these states are vibrationally cold. ${ }^{14}$ On this basis, we assign the IR spectra to singlet excited states of the tyrosine or phenylalanine chromophores.

The time evolution of the system in the excited state can be monitored by increasing the delay between UV-pump and IRprobe pulses, which produces changes in the IR spectrum (see Figure 4). Emergence of lines corresponding to the singlet excited state of tyrosine upon excitation of phenylalanine indicates that electronic energy migrates between these chromophores. At longer delays, broad features of a new state appear (see Figure S6). This broadening indicates that a significant part of the initial excitation is converted into vibrational energy. In this way, time-resolved IR spectroscopy reveals the fate of the electronic excitation. The excitation energy is initially deposited in the phenylalanine chromophore in its first singlet excited state. It then undergoes a nearly complete, nonradiative, singlet-singlet EET to the tyrosine chromophore, with the transfer rate being conformer-dependent. Finally, the tyrosine chromophore converts into a hot, long-lived state, from which the tyrosine side-chain loss occurs. ${ }^{14}$ The phenylalanine side-chain loss is not observed in our experiment because the energy transfer channel outcompetes the phenylalanine excited-state decay.

We select a band of the singlet excited state of phenylalanine at $3650 \mathrm{~cm}^{-1}$, which does not overlap with any vibrational transitions of the other excited states, to track its lifetime. The singlet excited-state lifetime of the phenylalanine chromophore in conformer $\mathrm{A}$ is $17 \pm 2 \mathrm{~ns}$ (Figure 4a), while that of conformer B is $9 \pm 2$ ns (Figure $4 \mathrm{~b}$ ).

It is illuminating to compare these values to the lifetime of the phenylalanine $S_{1}$ state in a peptide lacking tyrosine. A test experiment done on AcFAAK- $\mathrm{H}^{+}$has shown that the phenylalanine chromophore exhibits an $S_{1}$ decay constant of $83 \pm 3$ ns for conformer A and $88 \pm 5$ ns for conformer B (see Figure S7). This is consistent with results obtained on the other Phe-containing peptides. ${ }^{14}$ Assuming that the change in lifetime of the singlet excited state of the phenylalanine in the bichromophoric peptide is exclusively due to energy transfer to the acceptor chromophore, we derive the rate of energy transfer in the following way: $k_{\text {transfer }}=\frac{1}{\tau_{\text {AcFAYK- } \mathrm{H}^{+}}}-\frac{1}{\tau_{\text {AcFAKK- }}{ }^{+}}$; hence, $k_{\text {transfer }}$ (conformer A) $=(4.7 \pm 0.7) \times 10^{7} \mathrm{~s}^{-1}$ and $k_{\text {transfer }}($ conformer B $)=(10 \pm 2) \times 10^{7} \mathrm{~s}^{-1}$. Thus, the experiment provides the ratio of the energy transfer rates in the two conformers: $k_{\mathrm{B}} / k_{\mathrm{A}}=2.1 \pm 0.7$. With this value, we test the performance of two theoretical models for energy transfer. 
We compute the ratio of energy transfer rates with two different methods: Förster theory and Fermi golden rule theory based on electronic couplings between transition densities. The details of these models are described in the SI. The Förster approach is based on dipole-dipole interactions between transition dipole moments (TDMs) of the donor and acceptor chromophores, $^{29}$ and the quantum mechanical approach considers the full Coulomb interaction between 3D transition densities, accounting for electronic exchange and correlation effects, and orbital overlap contributions. ${ }^{30}$ The couplings are computed for three different truncated chromophores: model 1 corresponds to the minimal chromophore structure, model2 includes the local environment (i.e., part of the backbone), and model 3 includes the largest portion of the backbone (Figures S8-S10). Both TDMs and electronic couplings are computed with time-dependent density functional theory (TDDFT) $)^{31}$ and wave function-based equation-of-motion coupled cluster singles and doubles methods (EOM-CCSD). ${ }^{32}$

The results from the dipole-dipole approximation vary widely depending upon the fragmentation model and the electronic structure method employed. TDMs of the phenylalanine chromophore are governed by the local chromophore environment (Figure S12) owing to the highly polarizable phenylalanine chromophore. Also, higher-order multipoles become significant due to the short distance between chromophores $(\sim 12 \AA)$. These results are in line with previous work outlining the deficiencies of the dipole approximation. ${ }^{33,34}$

Computing the coupling based on transition densities reveals that the total electronic coupling values are strongly dominated by Coulomb contributions, with the exchange and orbital overlap effects being negligible (Tables $\mathrm{S} 1-\mathrm{S} 3$ in SI). For the intermediate and large fragmentation models (model 2 and model3), the TDDFT results (computed with two common density functional approximations: B3LYP $\mathrm{P}^{35,36}$ and CAM$\mathrm{B} \mathrm{LYP}^{37}$ ) deviate strongly, whereas EOM-CCSD is consistent with the experiment (Figure 5). Excited-state character analysis (Figures S13-S14) reveals that, instead of the fairly localized nature of the $\mathrm{L}_{\mathrm{b}}$ excitations predicted by wave function-based methods, the TDDFT excitations tend to spuriously delocalize from the chromophore toward the peptide backbone. This is the reason for the unbalanced description of the electronic couplings in the two chromophores. Furthermore, while the correlated wave function-based methods predict the two $\mathrm{L}_{b}$ excited states as the lowest in energy, in TDDFT, they do not necessarily coincide with the $S_{1}$ and $S_{2}$ states. Instead, they are embedded in the manifold of low-energy states, which increases the possibility of character mixing with the nearly degenerate excited states. The poor performance of TDDFT may be traced back to the lack of differential correlation effects, which are highly important for the description of $\mathrm{L}_{\mathrm{b}}$ states. ${ }^{38}$

To summarize, our experiment provides an alternative way of monitoring the EET and the subsequent photochemical processes. The low temperature in our ion trap allows measurement of conformer-specific infrared spectra of AcFAYK $-\mathrm{H}^{+}$. The relative orientation of the chromophores varies in the two conformers, while the distance between the chromophores does not change significantly. Comparing the IR spectrum of AcFAYK- $\mathrm{H}^{+}$with those of the two monochromophoric peptides $\left(\mathrm{AcFA}_{2} \mathrm{~K}-\mathrm{H}^{+}\right.$and $\left.\mathrm{AcA}_{2} \mathrm{YK}-\mathrm{H}^{+}\right)$reveals that only the $\mathrm{NH}$ and $\mathrm{OH}$ groups that are in the vicinity of the chromophores shift upon electronic excitation, confirming the peak assignments of the vibrational spectrum of the bichromophoric species. The UV-pump/IR-probe scheme

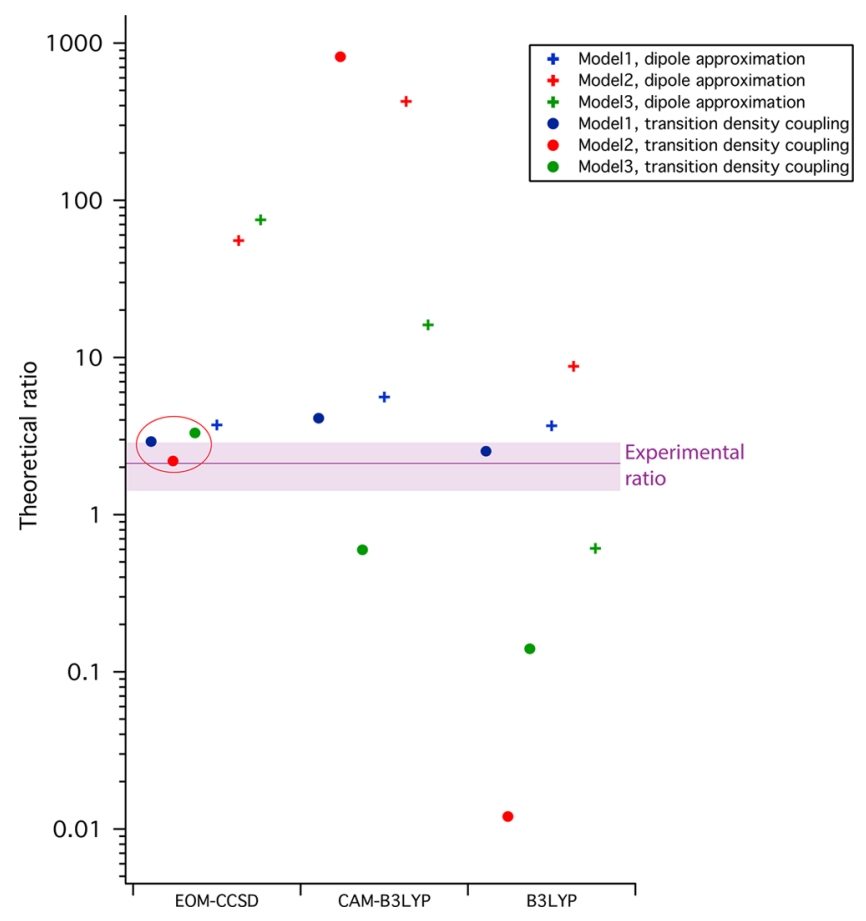

Figure 5. Ratio of EET rates $\left(k_{\mathrm{B}} / k_{\mathrm{A}}\right)$ predicted based on dipoledipole approximation and coupling of transition densities. The red oval highlights the transition density coupling computed with EOM-CCSD, which is in agreement with experiment for all cutting models. Notice the logarithmic vertical scale.

reveals the energy transfer processes that take place in real time after UV excitation of the phenylalanine or tyrosine chromophores in AcFAYK- $\mathrm{H}^{+}$. The measured singlet-singlet energy transfer rates vary by a factor of 2 for two different conformers. Carrying out the experiment in a cold ion trap narrows down the uncertainty caused by solvation and thermal vibrations and serves as a benchmark for determining the performance of the dipole-dipole approximation of the FRET model as well as that based on the coupling of transition densities. ${ }^{30}$ The dipole-dipole Förster approximation is insufficient for the system of interest because of the short distance between the chromophores. As for the quantum mechanical approach, couplings computed with EOM-CCSD are consistent with the experimental results, while those calculated with TDDFT using the B3LYP and CAM-B3LYP functionals are not. Dexter exchange of energy transfer is insignificant given that the exchange and the orbital overlap contribution to the total coupling are negligible.

The proposed technique of the cold ion pump-probe spectroscopy would benefit from shorter pulses of UV and IR lasers. One could envision applying the technique to a wider range of energy transfer phenomena using picosecond lasers in cold ion traps.

\section{ASSOCIATED CONTENT}

\section{S Supporting Information}

The Supporting Information is available free of charge on the ACS Publications website at DOI: 10.1021/acs.jpclett.8b01216.

Experimental and theoretical methods, spectroscopy of one-chromophore peptides, natural transition orbital analysis, time-resolved IR spectra, models of the chromophore truncation, transition dipole moments, and electronic couplings and their components. (PDF) 


\section{AUTHOR INFORMATION}

\section{Corresponding Author}

*E-mail: thomas.rizzo@epfl.ch.

\section{ORCID}

Clémence Corminboeuf: 0000-0001-7993-2879

Thomas R. Rizzo: 0000-0003-2796-905X

\section{Notes}

The authors declare no competing financial interest.

\section{ACKNOWLEDGMENTS}

T.R.R. and V.S. are grateful to the Swiss National Science Foundation (Grant Number 200020_165908) for the financial support of this work. All authors also thank the EPFL for its financial support. We thank Professor Ulrich Lorenz for helpful discussions.

\section{REFERENCES}

(1) Scholes, G. D.; Fleming, G. R.; Olaya-Castro, A.; van Grondelle, R. Lessons from Nature about Solar Light Harvesting. Nat. Chem. 2011, 3, 763-774.

(2) Tan, C.; Guo, L.; Ai, Y.; Li, J.; Wang, L.; Sancar, A.; Luo, Y.; Zhong, D. Direct Determination of Resonance Energy Transfer in Photolyase: Structural Alignment for the Functional State. J. Phys. Chem. A 2014, 118, 10522-10530.

(3) Chudakov, D. M.; Matz, M. V.; Lukyanov, S.; Lukyanov, K. A. Fluorescent Proteins and Their Applications in Imaging Living Cells and Tissues. Physiol. Rev. 2010, 90, 1103-1163.

(4) Feron, K.; Belcher, W. J.; Fell, C. J.; Dastoor, P. C. Organic Solar Cells: Understanding the Role of Förster Resonance Energy Transfer. Int. J. Mol. Sci. 2012, 13, 17019-17047.

(5) Huang, J.-S.; Goh, T.; Li, X.; Sfeir, M. Y.; Bielinski, E. A.; Tomasulo, S.; Lee, M. L.; Hazari, N.; Taylor, A. D. Polymer Bulk Heterojunction Solar Cells Employing Forster Resonance Energy Transfer. Nat. Photonics 2013, 7, 479-485.

(6) Chattoraj, M.; Bal, B.; Closs, G. L.; Levy, D. H. ConformationDependent Intramolecular Electronic Energy Transfer in a Molecular Beam. J. Phys. Chem. 1991, 95, 9666-9672.

(7) Danell, A. S.; Parks, J. H. FRET Measurements of Trapped Oligonucleotide Duplexes. Int. J. Mass Spectrom. 2003, 229, 35-45.

(8) Dashtiev, M.; Azov, V.; Frankevich, V.; Scharfenberg, L.; Zenobi, R. Clear Evidence of Fluorescence Resonance Energy Transfer in GasPhase Ions. J. Am. Soc. Mass Spectrom. 2005, 16, 1481-1487.

(9) Talbot, F. O.; Rullo, A.; Yao, H.; Jockusch, R. A. Fluorescence Resonance Energy Transfer in Gaseous, Mass-Selected Polyproline Peptides. J. Am. Chem. Soc. 2010, 132, 16156-16164.

(10) Daly, S.; Poussigue, F.; Simon, A.-L.; MacAleese, L.; Bertorelle, F.; Chirot, F.; Antoine, R.; Dugourd, P. Action-FRET: Probing the Molecular Conformation of Mass-Selected Gas-Phase Peptides with Förster Resonance Energy Transfer Detected by Acceptor-Specific Fragmentation. Anal. Chem. 2014, 86, 8798-8804.

(11) Rizzo, T. R.; Stearns, J. A.; Boyarkin, O. V. Spectroscopic Studies of Cold, Gas-Phase Biomolecular Ions. Int. Rev. Phys. Chem. 2009, 28, 481-515.

(12) Mališ, M.; Loquais, Y.; Gloaguen, E.; Biswal, H. S.; Piuzzi, F.; Tardivel, B.; Brenner, V.; Broquier, M.; Jouvet, C.; Mons, M.; et al. Unraveling the Mechanisms of Nonradiative Deactivation in Model Peptides Following Photoexcitation of a Phenylalanine Residue. J. Am. Chem. Soc. 2012, 134, 20340-20351.

(13) Hashimoto, T.; Takasu, Y.; Yamada, Y.; Ebata, T. Anomalous Conformer Dependent S1 Lifetime of 1-Phenylalanine. Chem. Phys. Lett. 2006, 421, 227-231.

(14) Zabuga, A. V.; Kamrath, M. Z.; Boyarkin, O. V.; Rizzo, T. R. Fragmentation Mechanism of UV-excited Peptides in the Gas Phase. J. Chem. Phys. 2014, 141, 154309.

(15) Loquais, Y.; Gloaguen, E.; Alauddin, M.; Brenner, V.; Tardivel, B.; Mons, M. On the Near UV Photophysics of a Phenylalanine
Residue: Conformation-Dependent $\pi \pi^{*}$ State Deactivation Revealed by Laser Spectroscopy of Isolated Neutral Dipeptides. Phys. Chem. Chem. Phys. 2014, 16, 22192-22200.

(16) Féraud, G.; Broquier, M.; Dedonder, C.; Jouvet, C.; Grégoire, G.; Soorkia, S. Excited State Dynamics of Protonated Phenylalanine and Tyrosine: Photo-Induced Reactions Following Electronic Excitation. J. Phys. Chem. A 2015, 119, 5914-5924.

(17) Soorkia, S.; Broquier, M.; Grégoire, G. Conformer- and ModeSpecific Excited State Lifetimes of Cold Protonated Tyrosine Ions. J. Phys. Chem. Lett. 2014, 5, 4349-4355.

(18) Malis, M.; Loquais, Y.; Gloaguen, E.; Jouvet, C.; Brenner, V.; Mons, M.; Ljubic, I.; Doslic, N. Non-Radiative Relaxation of UV Photoexcited Phenylalanine Residues: Probing the Role of Conical Intersections by Chemical Substitution. Phys. Chem. Chem. Phys. 2014, $16,2285-2288$.

(19) Hudgins, R. R.; Ratner, M. A.; Jarrold, M. F. Design of Helices That Are Stable in Vacuo. J. Am. Chem. Soc. 1998, 120, 12974-12975.

(20) Zabuga, A. V.; Rizzo, T. R. Capping Motif for Peptide Helix Formation. J. Phys. Chem. Lett. 2015, 6, 1504-1508.

(21) Stearns, J. A.; Boyarkin, O. V.; Rizzo, T. R. Spectroscopic Signatures of Gas-Phase Helices: Ac-Phe-(Ala)5-Lys-H+ and Ac-Phe(Ala)10-Lys-H+. J. Am. Chem. Soc. 2007, 129, 13820-13821.

(22) Hendricks, N. G.; Lareau, N. M.; Stow, S. M.; McLean, J. A.; Julian, R. R. Bond-Specific Dissociation Following Excitation Energy Transfer for Distance Constraint Determination in the Gas Phase. J. Am. Chem. Soc. 2014, 136, 13363-13370.

(23) Stearns, J. A.; Mercier, S.; Seaiby, C.; Guidi, M.; Boyarkin, O. V.; Rizzo, T. R. Conformation-Specific Spectroscopy and Photodissociation of Cold, Protonated Tyrosine and Phenylalanine. J. Am. Chem. Soc. 2007, 129, 11814-11820.

(24) Kopysov, V.; Boyarkin, O. V. Resonance Energy Transfer Relates the Gas-Phase Structure and Pharmacological Activity of Opioid Peptides. Angew. Chem., Int. Ed. 2016, 55, 689-692.

(25) Stearns, J. A.; Seaiby, C.; Boyarkin, O. V.; Rizzo, T. R. Spectroscopy and Conformational Preferences of Gas-Phase Helices. Phys. Chem. Chem. Phys. 2009, 11, 125-132.

(26) Voronina, L.; Masson, A.; Kamrath, M.; Schubert, F.; Clemmer, D.; Baldauf, C.; Rizzo, T. Conformations of Prolyl-Peptide Bonds in the Bradykinin 1-5 Fragment in Solution and in the Gas Phase. J. Am. Chem. Soc. 2016, 138, 9224-9233.

(27) Ebata, T.; Mizuochi, N.; Watanabe, T.; Mikami, N. OH Stretching Vibrations of Phenol- $(\mathrm{H} 2 \mathrm{O}) 1$ and Phenol- $(\mathrm{H} 2 \mathrm{O}) 3$ in the S1 State. J. Phys. Chem. 1996, 100, 546-550.

(28) Platt, J. R. Classification of Spectra of Cata-Condensed Hydrocarbons. J. Chem. Phys. 1949, 17, 484-495.

(29) Scholes, G. D. Long-Range Resonance Energy Transfer in Molecular Systems. Annu. Rev. Phys. Chem. 2003, 54, 57-87.

(30) Curutchet, C.; Mennucci, B. Quantum Chemical Studies of Light Harvesting. Chem. Rev. 2017, 117, 294-343.

(31) Furche, F.; Ahlrichs, R. Adiabatic Time-Dependent Density Functional Methods for Excited State Properties. J. Chem. Phys. 2002, $117,7433-7447$.

(32) Stanton, J. F.; Bartlett, R. J. The Equation of Motion CoupledCluster Method. A Systematic Biorthogonal Approach to Molecular Excitation Energies, Transition Probabilities, and Excited State Properties. J. Chem. Phys. 1993, 98, 7029-7039.

(33) Kenny, E. P.; Kassal, I. Benchmarking Calculations of Excitonic Couplings between Bacteriochlorophylls. J. Phys. Chem. B 2016, 120, $25-32$.

(34) Muñoz-Losa, A.; Curutchet, C.; Krueger, B. P.; Hartsell, L. R.; Mennucci, B. Fretting about FRET: Failure of the Ideal Dipole Approximation. Biophys. J. 2009, 96, 4779-4788.

(35) Becke, A. D. Density-Functional Thermochemistry. III. The Role of Exact Exchange. J. Chem. Phys. 1993, 98, 5648-5652.

(36) Lee, C.; Yang, W.; Parr, R. G. Development of the Colle-Salvetti Correlation-Energy Formula into a Functional of the Electron Density. Phys. Rev. B: Condens. Matter Mater. Phys. 1988, 37, 785-789. 
(37) Yanai, T.; Tew, D. P.; Handy, N. C. A New Hybrid ExchangeCorrelation Functional Using the Coulomb-Attenuating Method (CAM-B3LYP). Chem. Phys. Lett. 2004, 393, 51-57.

(38) Prlj, A.; Sandoval-Salinas, M. E.; Casanova, D.; Jacquemin, D.; Corminboeuf, C. Low-Lying $\pi \pi^{*}$ States of Heteroaromatic Molecules: A Challenge for Excited State Methods. J. Chem. Theory Comput. 2016, $12,2652-2660$. 